\title{
Classification of sets of stimuli with different stimulus characteristics and numerical properties'
}

\author{
SHIRO IMAI \\ THE JOHNS HOPKINS UNIVERSITY
}

The purpose of this experiment was to determine the effects of stimulus characteristics and numerical properties of sets of stimuli on classification. Sets contained 12 stimuli which were all identical, had different categories defined by color, or had different categories defined by attributes of color and size. Number of categories and numerical distribution of stimuli in categories were varied. For each set $S$ made a free classification and several restricted classifications in which the number of classes was specified. The results show: Sets of identical stimuli are classified into equal sized groups. Categorically defined stimuli are classified by category insofar as possible, but the tendency to numerical balance affects classifications with unbalanced numerical distributions or when the task restriction is incompatible with category classification. Stimuli defined by attributes are classified so as to maintain the attribute structure, although both category classification and numerical balance tendencies are evident with unbalanced numerical distributions and incompatible classification restrictions.

Many studies on classification have shown that the stimulus characteristics of sets of stimuli are highly relevant to classification performance (e.g., Shipstone, 1960; Imai \& Garner, 1965; Heidbreder, 1946; Shepard \& Chang, 1963; see Hunt, 1962, for more discussion). Typically, in such studies each possible stimulus occurs just once or, at least, all stimuli occur equally often. In general, however, a set of stimuli can have numerical properties of total number of stimuli, number of defined categories, and number of stimuli in each category, and these latter need not all be the same.

Since uniform numerical distributions of stimuli have been used on classification studies, it is not clear whether the classification performance is due to the actual stimulus characteristics or to the numerical balance of the stimulus categories, or to some combination of them.

The purpose of the present experiment was to investigate the effects of both stimulus characteristics and numerical properties of sets on clasşification. Different types of stimulus characteristics, different numerical distributions, and different types of classification restriction were used to determine the interactions between them.

\section{Subjects}

\section{METHOD}

A total of 60 male undergraduates was randomly selected from the elementary course in psychology at Johns Hopkins University. Each S participated singly in the one-hour experiment.

\section{Stimulus Sets}

Each set of stimuli consisted of 12 painted Plexiglas discs. Each stimulus was one of six colors (brown, yellow, green, blue, purple, or gray) and was either large or small ( 3.8 or $3.2 \mathrm{~cm}$ in diameter). Table 1 describes the 32 different stimulus sets used, which consisted of three basic types:

Uniform Sets. Set 1 consisted of 12 large stimuli of identical color, the actual color being different for different Ss.

Color Sets. Sets 2 through 22 consisted of 12 large stimuli with 2, 3, 4, or 6 different colors, and with different numerical distributions in the color-defined categories as indicated.

These sets were intended to provide categories defined on a nominal basis only, i.e., with no inherent structure to the stimulus property defining the categories. Color seemed the best property to use for this

Table 1. Stimulus Sets and Task Conditions

\begin{tabular}{|c|c|c|c|c|c|}
\hline Set & $\begin{array}{c}\text { Type } \\
\text { of } \\
\text { set }\end{array}$ & $\begin{array}{c}\text { No. of } \\
\text { stimulus } \\
\text { categories }\end{array}$ & $\begin{array}{l}\text { Numerical } \\
\text { distribution }\end{array}$ & $\begin{array}{l}\text { respe } \\
\text { req }\end{array}$ & $\begin{array}{l}\text { No. of } \\
\text { onse closses } \\
\text { quired }\left(N_{R}\right)\end{array}$ \\
\hline 1 & Uniform & 1 & 12 & free & 23456 \\
\hline 2 & Color & 2 & 102 & free & 234 \\
\hline 3 & 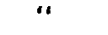 & “ & 93 & “ & "1" \\
\hline 4 & $\because$ & $" 1$ & 84 & $\because$ & " “ A \\
\hline 5 & " & $" 1$ & 75 & 11 & $\| 40$ \\
\hline 6 & " & $"$ & 66 & “ & "A A " \\
\hline 7 & $"$ & 3 & 1011 & $"$ & 23 \\
\hline 8 & 18 & 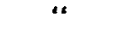 & 921 & $" 1$ & $" \cdots$ \\
\hline 9 & 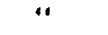 & $" 1$ & 822 & 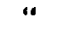 & " " \\
\hline 10 & "' & $" \prime$ & 651 & 11 & $" 1$ \\
\hline 11 & " & 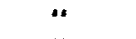 & 642 & $" 4$ & " . \\
\hline 12 & $"$ & $"$ & 444 & $“$ & " " \\
\hline 13 & $"$ & 4 & 9111 & $"$ & 234 \\
\hline 14 & $"$ & $" r$ & 8211 & $"$ & 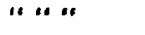 \\
\hline 15 & “ & “ & 6411 & $"$ & "1" \\
\hline 16 & " & $\because$ & 5421 & i. & " \\
\hline 17 & $"$ & “ & 4431 & $" \prime$ & 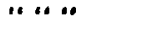 \\
\hline 18 & $\because$ & “. & 3333 & $" 1$ & " \\
\hline 19 & $“$ & 6 & 711111 & $" 4$ & 23456 \\
\hline 20 & $"$ & $"$ & 531111 & $"$ & 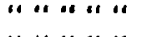 \\
\hline 21 & $"$ & $“$ & 333111 & $“$ & " “ «" \\
\hline 22 & $"$ & $" 1$ & 222222 & $" \prime$ & "114 \\
\hline 23 & $\begin{array}{r}\text { Color } \\
\times \text { Size }\end{array}$ & $2 \times 2$ & 9111 & $"$ & 234 \\
\hline 24 & " & $\because$ & 8211 & $“$ & "1 " \\
\hline 25 & $"$ & " & 6411 & $" 1$ & $" 11$ \\
\hline 26 & $"$ & $\because$ & 5421 & $"$ & $\|x\|$ \\
\hline 27 & “ & $\because$ & 4431 & $" 1$ & 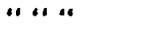 \\
\hline 28 & $\because$ & 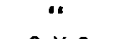 & 3333 & $" 1$ & " " \\
\hline 29 & $" 1$ & $3 \times 2$ & 711111 & 11 & 23456 \\
\hline 30 & $"$ & $“$ & 531111 & $" 1$ & " " " " " \\
\hline 31 & $“$ & $\because$ & 333111 & $" 1$ & “" “ " " " \\
\hline 32 & $"$ & “ & 222222 & $\because$ & " " " " \\
\hline
\end{tabular}


purpose. Nevertheless, the actual colors used for each numerical distribution were varied across ss to provide counterbalancing of colors with number of stimuli in a category. Analysis showed that no pair of colors was consistently grouped together in classification, so we can assume that these are nominally defined categories. Therefore, all results will be presented without regard to the actual colors used.

Color X Size Sets. Sets 23 through 32 consisted of four or six categories defined by orthogonal combinations of 2 or 3 colors and 2 sizes. These sets had categories in which the defining stimulus properties do have a structure, and the categories are not just nominal. Rather, they are defined by perceived attributes of the stimuli, and the stimuli could differ in size, color, or both color and size.

Since the purpose of using these sets was to determine whether the nature of the stimulus properties which define the categories affects classification performance, these sets all have numerical distributions and classification restrictions identical to the equivalent sets with categories defined by just color (i.e., Sets 23-28 are the same as Sets 13-18 and Sets 29-32 are the same as Sets 19-22)。

The assignment of number of stimuli to a particular category was counterbalanced across Ss. For these stimulus sets, however, all categories are not equivalent, as will become clear from the results. Thus counterbalancing of number with actual category is necessary with these sets.

Tasks

Free classification. The first classification made by $S$ for each set of stimuli was a free classification (Imai \& Garner, 1965) in which neither the number of classes nor the numerical distribution within classes was specified by $E$. $S$ was simply required to form at least two classes.

Restricted classification. After the free classification, E randomly rearranged the stimuli and then required $S$ to make the various restricted classifications indicated in Table 1. The numbers of classes, the only restriction, were required from $\mathrm{E}$ in random order for each set, but all classifications were completed for one set before another set was presented.

Procedures. Before starting the experiment, E informed $S$ of the general nature of the experiment. He also informed $S$ that there were no right or wrong methods of classification, other than with respect to the number of classes required.

The stimuli from each set were placed on a table in front of $\mathrm{S}$, in a more-or-less haphazard arrangement. $S$ was allowed to form his classes either by separating the stimuli or by arranging them in piles, or in any other manner as long as it was clear to $\mathrm{E}$ which stimuli formed a class.

The 32 stimulus sets were presented to different Ss in different orders, randomly determined except

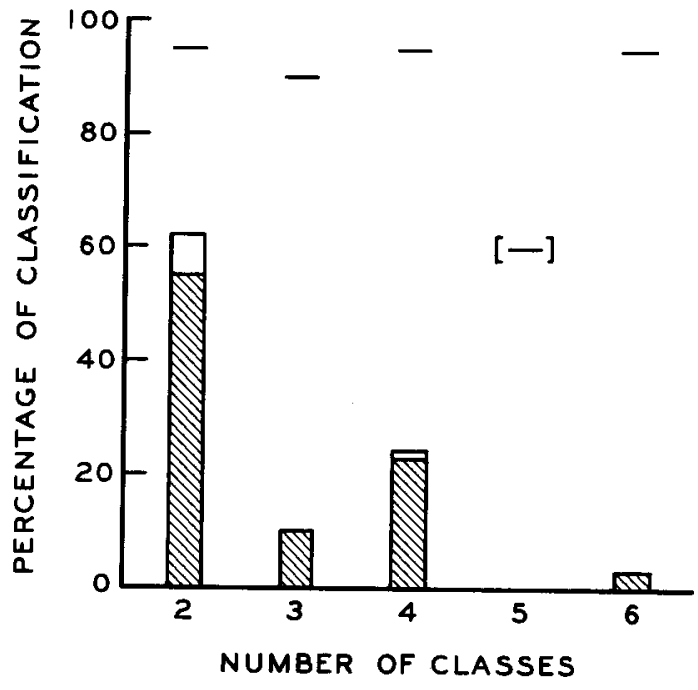

Fig. 1. Classification of Uniform Sets by number of classes. The vertical bars show the percentage of Ss who used each of the numbers of classes in free classification, and the shaded portion shows the percentage of classifications which were numerically balanced. The horizontal lines show the percentage of classifications which were numerically balanced when the number of classes was required in restricted classification.

that different numbers of categories were spread reasonably uniformly through the series of stimulus sets.

\section{UNIFORM SETS}

The only definable property of the uniform sets is total number of stimuli. Therefore classification of these sets provides a basis of comparison when differentiating stimulus properties are introduced into the stimulus sets, since the only basis on which classification can occur with uniform sets is the numerical distributional preferences of $S$.

Numerical balance. The results of both free and restricted classification of Uniform Sets are shown in Fig. 1. In free classification, two classes were used most frequently, and four classes next most frequently. Furthermore, as shown by the shaded part of the vertical bars, most of the classifications (91\%) resulted in a numerical balance in that each of the classes had an equal number of members. It is interesting that five classes were never used in free classification, in that five classes cannot result in a numerical balance with a total of 12 stimuli.

When $E$ specified the number of classes to be formed, again the typical result is that $S$ put an equal number of stimuli in each class. Excluding the impossible case where $\mathrm{N}_{R}=5,94 \%$ of the restricted classifications resulted in numerical balance, and even when five classes are required, $60 \%$ of the Ss (shown as a numerical balance result in Fig. 1) used a classification of $2-2-2-3-3$, which is the closest possible approximation to a numerical balance. 
So with no differentiating stimulus characteristics, Ss use a small number of stimulus classes, and, more importantly, produce classes of equal size.

\section{COLOR SETS}

When each stimulus in a set has a color which specifies a stimulus category for it, the expected type of classification is for $S$ to form one class for each category-and when $S$ does this we shall use the term category classification. Such classification is at the concrete or perceptual level (Bruner et al.s 1956, p. 9). Since, however, Ss have numerical preferences which involve both the number of classes and the numerical balance in these classes, we might expect there to be some interaction between the categorical and the numerical properties of the sets in determining classification behavior.

\section{Free Classification}

Category classification. Figure 2 shows the types of classification used in free classification as a function of the number of stimulus categories, averaged over all distributions within each number of categories. For each number of stimulus categories the modal classification was to use an equal number of stimulus classes. Furthermore, a substantial majority of all classifications $(70 \%)$ were classifications by color, or category classification.

Numerical preferences. Even though so many of the classifications are by color category, the effect of S's numerical preferences is still quite evident. In Fig. 2, for example, there is a fairly regular decrease in the percentage of color classifications as the number of stimulus categories is increased, and Ss use the preferred smaller number of classes more frequently. Thus as the number of stimulus categories becomes too much greater than the preferred number of categories, various categories will be combined to give a smaller number of classes.

Still further effects of numerical preferences are shown in Fig. 3, where the percentage of color classi- fications is shown as a function of the numerical distribution. For this figure, Sets $2,7,13$, and 19 were considered extremely unbalanced, Sets $6,12,18$, and 22 as balanced, and the rest of the Color Sets as unbalanced. This figure shows that there are fewer color classifications with unbalanced sets and even fewer with extremely unbalanced sets.

Of all non-category classifications for the Color Sets, $56 \%$ showed an exact numerical balance. This percentage is very high considering that some of the sets cannot allow numerically balanced classification without splitting of a single category, because one category has more than half the total number of stimuli. Compatible Restricted Classification

When classification is restricted by specification of the number of stimulus classes, the restriction may be compatible with the number of stimulus categories (and thus with the preferred type of classification) or may be incompatible. Each type of restriction will be examined separately.

Compatible category classification. When the restriction is compatible with category classification, that type of classification is very frequently used, as shown in Fig. 2. Even with restricted classification, however, there is some decreased use of category classification with the larger numbers of categories as occurred with free classification. But this decrease is less than with free classification, with the result that free and compatible restricted classifications become less alike with the larger number of stimulus categories.

Numerical balance tendency. Again as with free classification, the number of category classifications decreases with more unbalanced stimulus distributions, as shown in Fig. 3. In this case $65 \%$ of all non-category classifications showed an exact numerical balance, an even greater percentage than with free classification. Incompatible Restricted Classification

When the number of classes required by $\mathrm{E}$ is not the same as the number of stimulus categories, the

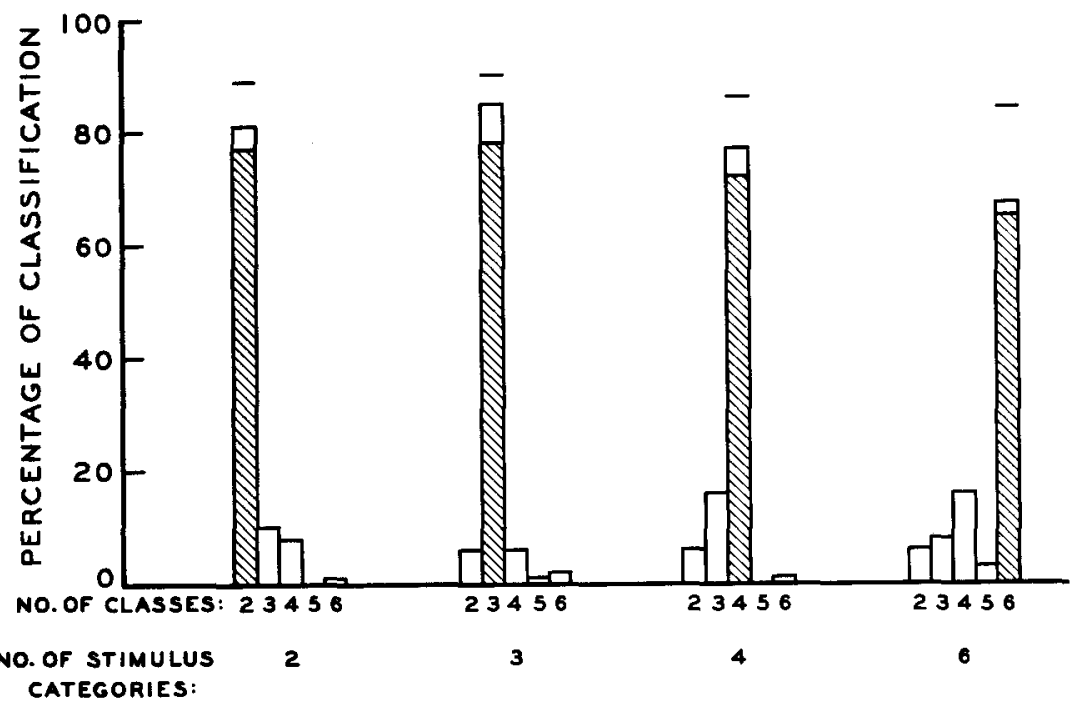

Fig. 2. Classification of Color Sets by number of classes and number of stimulus categories. The vertical bars show the percentage of Ss who used each number of classes with each number of stimulus categories, averaged for all numerical distributions, in free classification. The shaded portions of each bar show the percentage of color classification. The horizontal lines show the percentage of color classification in restricted classification when the required number of classes was the same as the number of stimulus categories. 


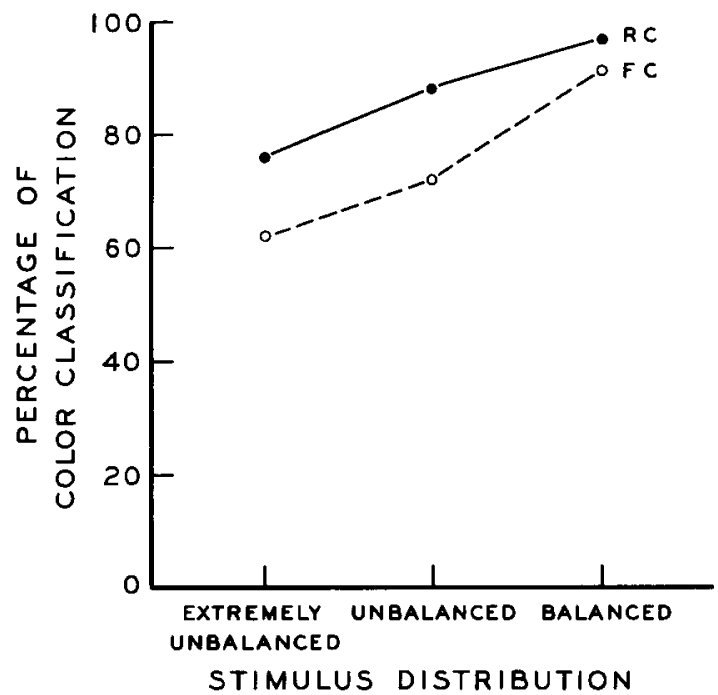

Fig. 3. Percentage of color classification of Color Sets with free classification (FC) and compatible restricted classification (RC) as a function of the numerical distribution.

required number may be greater or less than the number of stimulus categories.

Too few classes. With three stimulus categories, but $\mathrm{N}_{\mathrm{R}}=2,72 \%$ of $\mathrm{Ss}$ (for Sets from 7 through 11 ) grouped the two smallest categories together to form the two required classes, thus tending toward numerical balance. However, with Set 12, having a balanced distribution, the majority (53\%) actually split one category to form two classes of four of one color and two of another. Thus in this case the preference for numerical balance actually produced a category split-a very uncommon type of classification.

With four stimulus categories and $\mathrm{N}_{\mathrm{R}}=3$, the modal classification $(60 \%)$ was again to group the two smallest categories together, and in this case there was no exception for the balanced Set 18. With four stimulus categories and $\mathrm{N}_{\mathrm{R}}=2$, the modal type of classification (72\%) was to group the three smallest categories together for Sets 13,14, and 15, and to group the largest and the smallest together and the remaining two categories together for Sets 16,17 , and $18(62 \%)$-in each case classifications which came closest to providing numerical balance.

Similarly, with six stimulus categories, Ss $(62 \%)$ grouped stimulus categories without splitting to get the required number of classes and to get the best numerical balance. The only exception was classification of Set 20 into two classes, in which case the most frequent single classification (30\%) was to keep the largest category separate instead of combining it with one of the smallest categories.

Too many classes. The only cases where more classes were required than there were stimulus categories were with two stimulus categories, with three and with four required classes. With three required classes, the modal classification (57\%) was splitting of the largest category into two even sized subclasses. An exception occurred with balanced Set 6 , where the even split of one category produces a large numerical imbalance. In this case, the modal type of classification (33\%) involved formation of a third group by drawing two stimuli from each of the two categories.

With $\mathrm{N}_{\mathrm{R}}=4,58 \%$ of the classifications involved even subdivision of stimuli in each of the two categories to form four classes. An exception occurred with Set 3, where the largest category was split into three classes of three stimuli each, which with the other category of three stimuli provided complete numerical balance.

\section{Summary}

With sets of stimuli consisting of nominal categories, the preferred type of classification consists of forming one class with each category. However, if the stimulus set has too many categories or too unbalanced a numerical distribution, the classification tends to be modified to provide a smaller number of classes and a numerically balanced distribution of stimuli in the classes, even if categories must be combined or split. When category classification is made impossible by requiring a number of classes different from the number of categories, these numerical preferences assume an even more important role. Thus classification is not simply a function of the properties of the stimuli which define the categories; it is also a function of the numerical properties of these categories.

\section{COLOR $X$ SIZE SETS}

When a category for a stimulus is specified by two attributes, color and size in this case, there are more types of classification which are directly related to the stimulus properties than when stimuli are specified only by nominal categorization. We can still obtain category classification, in which case $S$ disregards the means by which the categories are formed. In addition, however, we can obtain attribute classifica$t_{i o n}$ in which stimuli are grouped so as to provide complete differentiation with respect to one attribute, and complete generalization with regard to the other attribute, in that within each class formed all levels of the second attribute exist. Such classification maintains the attribute structure, and is at the abstract level.

\section{Free Classificafion}

Category classification. Figure 4 shows the percentages of category (color $\mathrm{X}$ size) classifications made for the two different numbers of categories, averaged over all numerical distributions. Even with stimuli whose categories are formed by two attributes, a substantial number of the classifications are of the category type.

If the means by which categories are formed has no effect on category classification, these percentages should be the same as for the comparable conditions in Fig. 2. With Color Sets, four or six stimulus categories, $68 \%$ of all classifications are by category, while this percentage drops to 41 with the Color $\mathbf{X}$ Size Sets. Thus the nature of the underlying property defining the categories does have a substantial effect on the use of category classification. 
Attribute classification. The major reason for the decrease in category classification with Color XSize Sets lies in the substantial use of one or another form of attribute classification. Altogether, $42 \%$ of all classifications are attribute classifications, with $35 \%$ of these being classification by color, and only $7 \%$ being classification by size. Thus there does appear to be a preference for color as the differentiating attribute, but we do not know whether it is a preference based on discriminability or not (Imai \& Garner, 1965).

Attribute or numerical preference? With Color Sets, there was a decreased amount of category classification with the larger numbers of stimulus categories. The same result holds with Color $X$ Size Sets, even to the extent that category classification is used less than half the time with sets of six categories. What is used in place of category classification with these sets is attribute classification. Attribute classification, however, does have the additional property that fewer total classes are used, and we saw that Ss prefer a small number of classes when classifying Uniform Sets. Thus we cannot be sure whether the great use of attribute classification with the Color XSize is due primarily to a preference to classify by attribute, or to a preference for a smaller number of classes. These two preferences are not in conflict, while there is conflict between a small number of classes and category classification.

Numerical balance tendency. Another numerical factor in classification involves a preference for numerical balance, and this preference may be in conflict with either attribute or category classification with unbalanced numerical distributions. Figure 5 shows the percentage of each type of classification as a function of numerical distribution, where Sets 23 and 29 are considered extremely unbalanced, Sets 28 and 32 as balanced, and the rest as unbalanced. As with Color Sets; there is a decreased use of classification by stimulus property with unbalanced distributions. And a large percentage $(30 \%)$ of all classifications which are neither attribute nor category classifications do show an exact numerical balance. This percentage is smaller than in the case of Color Sets, but still substantial considering the number of sets where numerical balance is impossible without splitting stimulus categories.

\section{Compatible Restricted Classification}

Restricted classification with Color X Size Sets makes possible compatibility with category classification, compatibility with attribute classification, and incompatibility with either of these.

Compatible category classification. The horizontal lines in Fig. 4 show the percentage of times that category (color $\mathrm{X}$ size) classification was used when four or six classes were required with the same number of stimulus categories. These percentages are quite high and very nearly the same as the comparable percentages obtained with Color Sets, shown in Fig. 2. Thus when task restriction is compatible with category classification, Ss are quite able and willing to use it, even when it is not the preferred type of classification.

Compatible attribute classification. Similarly high percentages are obtained when the task restriction is compatible with attribute classification. With $2 \times 2$ sets, and $\mathrm{N}_{\mathrm{R}}=2$, color classification is used $73 \%$ of the time and size classification $21 \%$ of the time, for a total of $94 \%$, averaged over all numerical distributions. With $3 \times 2$ sets, $N_{R}=3$, color classification is used $87 \%$ of the time, and when $\mathrm{N}_{\mathrm{R}}=2$, size classification is used $66 \%$ of the time. Thus it is clear that either category or attribute classification can be carried out fairly easily, so that the differences in type of classification in free classification must represent preference for the type of classification rather than difference in difficulty of classification.

Attribute or numerical preference? With free classification we noted that the high use of attribute classification was consistent with S's preference for a small number of classes, and thus that we could not be sure that there existed a pref-

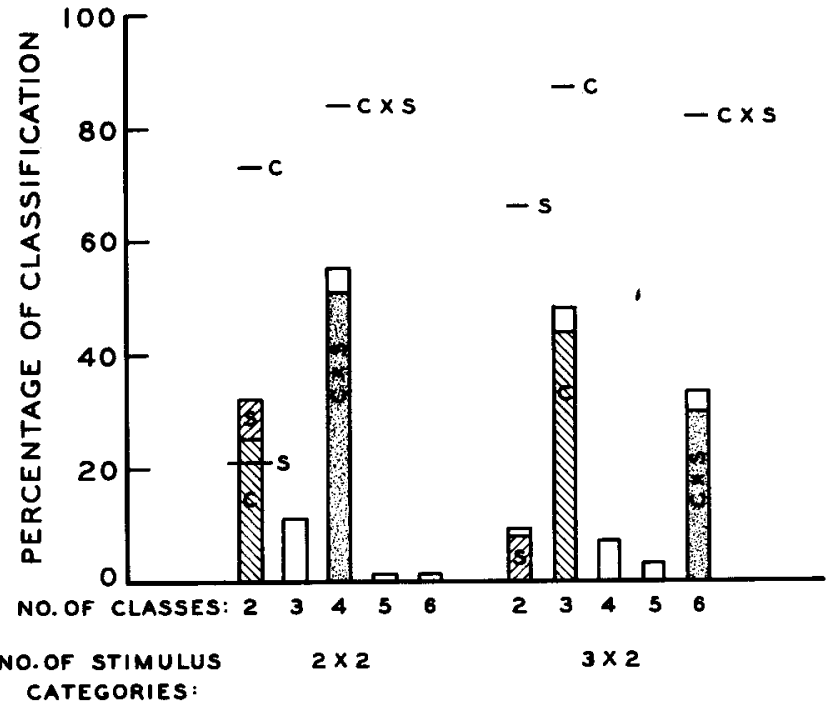

Fig. 4. Classification of Color $x$ size Sets by number of classes and number of stimulus categories. The vertical bars show the percentage of Ss who used each number of classes with each number of stimulus categories, averaged for all numerical distributions, in free classification. The shaded part of each vertical bar shows the percentages of color classification (C), size classification (S), and color $X$ size classification (C X S) The horizontal lines show these percentages when the indicated number of classes was required in restricted classification. 
erence for attribute classification per se. Furthermore, with unbalanced numerical distributions there was a decreased use of attribute classification, so that we know that numerical preferences are still operative with these sets of stimuli. Comparison between Color Sets and Color $X$ Size Sets with restricted classification can help clarify this problem.

Attribute classification for the two balanced Color $\mathrm{X}$ Size Sets (Sets 28 and 32) occurred $90 \%$ of the time when the required number of classes was compatible with attribute classification. If we look at the comparable Color Sets (Sets 18 and 22), and note the classifications which have the same numerical properties as those with the Color X Size Sets, given the same task restriction, we find that $81 \%$ of these classifications are the equivalent of attribute classifications. The fact that this percentage is higher when attributes actually exist, rather than just categories, in itself suggests that the existence of attributes does influence the nature of the classification.

This difference is much greater with unbalanced sets, where the non-preferred unbalanced distributions can interfere with attribute classification. Sets 23 and 29 are the most unbalanced of the Color X Size Sets, yet $78 \%$ of all classifications were of the attribute type, and only $5 \%$ of these classifications produced the best possible numerical balance. The comparable Color Sets are 13 and 19 , and with these sets, and the same classification restrictions, $68 \%$ of the classifications resulted in the best possible numerical balance.

Thus it is clear that there is a definite preference for attribute classification which exists independently of preferences for small numbers of classes and numerical balance in these classes.

Numerical balance tendency. Nevertheless, the preference for numerical balance still does exert some influence on the classifications, as indicated in Fig. 5 . Even with restricted classification, the unbalanced distributions show fewer attribute or category classifications than do the balanced distributions. And $45 \%$ of the classifications which were not category or attribute classifications did show an exact numerical balance.

\section{Incompatible Restricted Classification}

There are few incompatible restrictions with the Color X Size Sets because of the number of ways in which the restriction can be compatible. Nevertheless, examination of classifications with incompatible restrictions does clarify and strengthen conclusions which are apparent with free and compatible restricted classification. In particular, we will examine data with $2 \times 2$ sets, and $N_{R}=3$, the only incompatible restriction used with these sets.

Relative preference for attribute. It is clear that attribute classification is a highly preferred type of classification, and furthermore that classification with color as the differentiating attribute is preferred to the use of size as the differentiating attribute, although we cannot know why color is the preferred attribute.
Nevertheless, this same preference is evident in classifications with incompatible restriction.

Ss rarely subdivide stimulus categories in their classifications unless the classification restriction forces them to do so, regardless of the means by which the categories are formed. When four categories are formed from two dichotomous attributes, these categories can be combined in meaningfully different ways. And when it is required that three classes must be formed, then two of the categories must be combined to reduce the four categories to three classes.

Two basic methods of combining categories produce partial generalization with respect to one attribute. For example, if large blue is combined with small blue, leaving large yellow and small yellow intact, then partial generalization with respect to size has occurred, because both sizes exist within one color. If partial generalization occurs with respect to size, then maximum differentiation also occurs with size, rather than with color. In the above illustration each of the three classes differs with respect to size for some or all of the elements in each class, but two of the classes do not differ from one another with respect to color. Therefore, if color is the preferred differentiating attribute, we should expect partial generalization to occur with color when the classification restriction forces two categories to be combined.

For all distributions (Sets 23-28), $48 \%$ of the classifications produced partial generalization with respect to color, compared to $37 \%$ for size thus confirming the preference for color as the differentiating attribute. With just balanced Set 28 , with no numerical factors operating, $63 \%$ of the classifications produced partial generalization with respect to color.

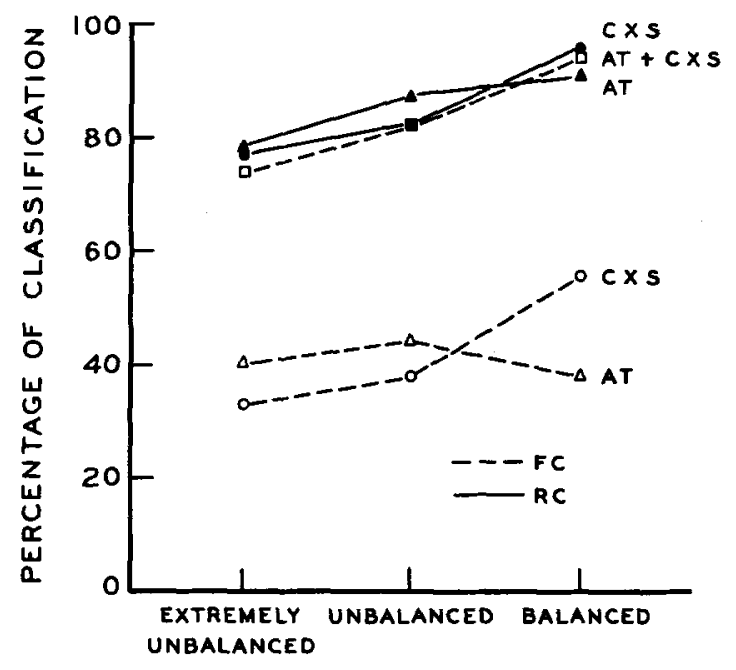

\section{STIMULUS DISTRIBUTION}

Fig. 5. Classification of Color $x$ Size Sets as a function of numerical distribution. The dashed lines show percentage of attribute classification (AT), either color or size, and color $\mathbf{X}$ size classification (C X S), and the sum of these in free classification (FC). The solid lines show the same types of classification with compatible restricted classification ( $R C$ ). 
Attribute vs numerical balance.

There is another basic method of combining categories which does not preserve attribute structure. For example, we can combine four categories into two by combining large yellow with small blue, and small yellow with large blue. Now both attributes differentiate and generalize, and both attributes are correlated within each class. Such classifications almost never occur in free or restricted classification, a fact which in itself makes the important role of attribute clear.

An equivalent type of classification can occur when three classes must be formed from four categories, as in the present case, by combining two categories to provide correlated attributes within the combined class. For example, we can combine large yellow with small blue, using small yellow and large blue as the other two classes. Again, such classifications are rarely used. There is one case, however, in which an exact numerical balance can be produced if $S$ will use such a classification, while an unbalanced distribution will be produced if attribute structure is maintained by using partial generalization as the classification strategy. Set 27 has a 4-4-3-1 distribution, and these frequencies were counterbalanced across subjects with respect to the combination of category and frequency. The result of the counterbalancing was that $20 \mathrm{Ss}$ had the frequencies of 3 and 1 assigned so that if they were combined a correlated subclass would be produced along with exact numerical balance. Not a single $S$ used that strategy, but all 20 Ss used partial generalization with respect to either color or size. Thus when the preference for numerical balance is put in direct conflict with the preference for attribute classification, the latter preference dominates the behavior.

Nevertheless, with very unbalanced distributions, the preference for numerical balance is still operative. To illustrate, with Set 23, any two of the categories could be combined, and if Ss were unconcerned with numerical distributions, all combinations would occur equally often, since each category was assigned the highest frequency equally often. Of the four combinations which produce partial generalization, half of them involved any one category. However, only $32 \%$ of the classifications involved combinations with the most frequent category, and half of these occurred with combinations of the two small stimuli-a fact which suggests that total physical size of groups had some effect, and which may be the explanation for the preference of color over size as the differentiating áttribute.

\section{SUMMARY}

Numerical preferences exist in classification, and these are shown with classification of sets of identical stimuli as a preference for a small number of classes and equal sized classes (numerical balance).

Category classification, in which one class is formed with each stimulus category, is preferred when only a nominal categorization exists for the stimuli. However, the existence of numerical preferences is demonstrated as well. With free classification, a smaller number of classes than categories will be used if there are too many categories, or if the numerical distributions are too unbalanced, and such classifications will tend toward numerical balance. With compatible restricted classification there is no choice concerning the number of classes, but combinations will be formed which tend toward numerical balance if the numerical distribution is too unbalanced. With incompatible restricted classifications, where category classification is impossible, categories are combined or split to produce numerical balance.

Attribute classification, in which classes are differentiated with respect to one attribute and generalized with respect to another, occurs when stimulus categories are formed with orthogonal combinations of attributes. Category classification still is frequently used in free classification, and nearly always when the restriction is compatible with such classification. Numerical factors can still be shown to operate, however, when stimulus distributions are very unbalanced. Nevertheless, within wide limits the preference for attribute classification will dominate the preference for numerical balance, even under conditions of classification restriction which is incompatible with simple attribute classification.

\section{References}

Bruner, J. S., Goodnow, J. J., \& Austin, G. A. A study of thinking. New York: John Wiley, 1956.

Heidbreder, E. The attainment of concepts: II. The problem. J. gen. Psychol., 1946, 35, 191-223.

Hunt, E. B. Concept learning. New York: John Wiley, 1962. Imai, S., \& Garner, W. R. Discriminability and preference for attributes as factors in free and constrained classification. J. exp. Psychol., 1965, 69, 596-608.

Shepard, R. N., \& Chang, J. J. Stimulus generalization in the learning of classifications. J. exp. Psychol., 1963, 65, 94-102. Shipstone, E. I. Some variables affecting pattern conception. Psychol. Monogr., 1960, 74, No. 17.

\section{Note}

1. This study is based upon a dissertation submitted to The Johns Hopkins University in partial fulfillment of the requirements for the $\mathrm{PhD}$ degree. The author would like to express particular appreciation to Wendell R. Garner for his helpful guidance. This study was done in part under Contract Nonr-248(55) and in part under Contract Nonr-4010(03) between the Office of Naval Research and The Johns Hopkins University. This is Report No. 13 under the latter contract. Reproduction in whole or in part is permitted for any purpose of the United States Government. 